\title{
Differentiation of HL-60 cells in serum-free hematopoietic cell media enhances the production of neutrophil extracellular traps
}

\author{
YUN GUO $^{1 *}$, FEI GAO $^{2 *}$, QIAN WANG $^{1}$, KANG WANG $^{3}$, SHANSHAN PAN $^{1}$, \\ ZHENZHEN PAN ${ }^{1}$, SHIYAO XU ${ }^{1}$, LING LI $^{1}$ and DEYU ZHAO ${ }^{4}$ \\ ${ }^{1}$ Department of Respiratory Medicine, The Affiliated Wuxi Children's Hospital of Nanjing Medical University; \\ ${ }^{2}$ Department of Intensive Care Unit, Wuxi People's Hospital Affiliated to Nanjing Medical University; \\ ${ }^{3}$ Department of Laboratory, The Affiliated Wuxi Children's Hospital of Nanjing Medical University, Wuxi, Jiangsu 214000; \\ ${ }^{4}$ Department of Respiratory Medicine, Children's Hospital of Nanjing Medical University, Nanjing, Jiangsu 210000, P.R. China
}

Received April 17, 2020; Accepted October 28, 2020

DOI: 10.3892/etm.2021.9784

\begin{abstract}
Neutrophil extracellular traps (NETs) are web-like structures made of chromatin and have been identified to have a role in the host's immune defense. Differentiated human promyelocytic leukemia HL-60 cells (dHL-60) have been used to study the mechanisms of NETs formation, as neutrophils have a short lifespan that limits their use. However, dHL-60 cells are inefficient at generating NETs and therefore are not ideal replacements for neutrophils in studying of NET formation. In the present study, the optimal cell culture conditions and differentiation time that result in the most effective release of NETs from dHL-60 cells upon stimulation were determined. HL-60 cells were cultured in serum (FBS) or serum-free (X-VIVO) medium and differentiated using all-trans retinoic acid (ATRA) or dimethyl sulfoxide (DMSO). dHL-60 cells were stimulated with phorbol 12-myristate 13-acetate (PMA) or $\mathrm{Ca}^{2+}$ ionophore (CI). Cell differentiation and apoptosis, as well as the formation of reactive oxygen species (ROS) and citrullinated histone $\mathrm{H} 3$ (citH3) were analyzed using flow cytometry. NETs were visualized using fluorescence microscopy and NET quantification was performed using PicoGreen. Induction of HL-60 cells for five days produced
\end{abstract}

Correspondence to: Dr Ling Li, Department of Respiratory Medicine, The Affiliated Wuxi Children's Hospital of Nanjing Medical University, 299 Qingyang Road, Liangxi, Wuxi, Jiangsu 214000, P.R. China

E-mail: liling@njmu.edu.cn

Dr Deyu Zhao, Department of Respiratory Medicine, Children's Hospital of Nanjing Medical University, 72 Guangzhou Road, Gulou, Nanjing, Jiangsu 210000, P.R. China

E-mail: zhaodeyu988@126.com

${ }^{*}$ Contributed equally

Key words: HL-60, neutrophil, neutrophil extracellular traps, all-trans retinoic acid, dimethyl sulfoxide, reactive oxygen species, histone citrullination, serum-free medium, cell differentiation the best results in terms of differentiation markers and cell viability. Both ATRA- and DMSO-induced dHL-60 cells were able to release NETs upon PMA and CI stimulation; dHL-60 cells in serum-free medium produced more NETs than those in serum-containing medium. DMSO-dHL-60 (X-VIVO) cells were most efficient at producing NETs and ROS upon stimulation with PMA, while ATRA-dHL-60 (X-VIVO) cells were most efficient at producing NETs and citH3 upon stimulation with CI. It was concluded that DMSO-dHL-60 (X-VIVO) may be a model for the study of ROS-high NETosis and ATRA-dHL-60 (X-VIVO) may be suitable for ROS-low NETosis.

\section{Introduction}

Neutrophils are the most abundant type of leukocyte in the human bloodstream and are recruited from the circulation to sites of infection, inflammation or damage (1). As key components of the innate immune system, neutrophils have various functions, such as releasing granule proteins, generating reactive oxygen species (ROS) and releasing neutrophil extracellular traps (NETs) $(1,2)$.

NETs are web-like structures made of chromatin and are decorated with histones, myeloperoxidase (MPO), neutrophil elastase and cathepsin G. NETs are known for their ability to defend against pathogens, including Staphylococcus aureus (3), pneumococci (4) and streptococci (5). Previous studies $(3,5,6)$ have highlighted the 'double-edged sword' concept, as NETs have been identified to have a role in the host immune defense, while also being implicated in the pathophysiology of various diseases, including thrombosis. A further understanding of how neutrophils cast their NETs is critical for exploring the pathogenesis of diseases associated with NETs.

Numerous pathogens may induce neutrophils to form NETs via different signals, such as Toll-like receptors, Fc receptors, chemokine receptors and cytokine receptors (1). Brinkmann et al (7) first described the NETosis pathway in 2004; this cell death process depends on the generation of ROS by NADPH oxidase (8) and the generation of citrullinated histone $\mathrm{H} 3$ (citH3) by peptidylarginine deiminase type 4 (PADI4) (9). Neutrophils are also able to release 
NETs in a cell death-independent manner called vital NETosis. In this process, NETs are released rapidly and NADPH oxidase function is not necessary $(10,11)$. Recently, Chen et al (12) reported that cytosolic lipopolysaccharide or cytosolic gram-negative bacteria are able to activate the noncanonical (caspase-4/11) inflammasome and trigger gasdermin D-dependent neutrophil cell death recently called noncanonical NETosis, which is still debatable. The involvement of a number of pathways implies that the progress of NET formation is complex; however, the short life of neutrophils makes it difficult to explore the molecular and cellular mechanisms of NET formation.

HL-60 cells may be differentiated into granulocyte-like cells using all-trans retinoic acid (ATRA) or dimethyl sulfoxide (DMSO). A model based on a stable cell line has been used to reduce dissonant results due to different experimental settings or conditions (13-15) and differentiated HL-60 (dHL-60) cells have been used to study neutrophil functions, including NETosis (16-18). However, the inefficiency of dHL-60 cells in generating NETs makes it challenging to completely replace neutrophils in the analysis of NET formation. In the present study, the cell culture and differentiation conditions that lead to the most effective release of NETs from dHL-60 were optimized.

\section{Methods}

Reagents. Fetal bovine serum (FBS), goat sera, RPMI-1640 medium and PBS were purchased from Biological Industries Israel Beit Haemek. X-VIVO ${ }^{\text {TM }} 15$ medium was purchased from Lonza (cat.no.04-418Q).FITC Annexin V Apoptosis Detection kits were purchased from BD Pharmingen (cat. no. 556547). Anti-CD11b-PE, anti-CD16-APC and anti-CD66b-FITC were purchased from Biolegend (cat. no. 101207, 302011 and 305104, respectively). ATRA was purchased from MedChemExpress (cat. no. 302-79-4). DMSO was purchased from Sigma-Aldrich (Merck KGaA; cat. no. D8418-100). Neutrophil isolation kits were purchased from TBD (cat. no. LZS11131). citH3 antibody (histone $\mathrm{H} 3$ citrulline $\mathrm{R} 2+\mathrm{R} 8+\mathrm{R} 7$ ), anti-MPO antibody and $\mathrm{Ca}^{2+}$ ionophore (CI) were purchased from Abcam (cat. nos. ab5103, ab9535 and ab120287, respectively). Goat anti-mouse Alexa Fluor 488, ProLong Gold Antifade reagent and histone $\mathrm{H} 3$ mouse antibody were purchased from Cell Signaling Technology, Inc. (cat. nos. 4408s, 9071S and 14269s, respectively). Goat anti-rabbit Alexa Fluor 568, Total ROS Assay kits and Quant-iT ${ }^{\mathrm{TM}}$ PicoGreen $^{\text {TM }}$ double-strand (ds)DNA Assay kits were purchased from Thermo Fisher Scientific, Inc. (cat. no. A-11011, 88-5930-74 and P7589, respectively). R-PE-antibody conjugation kits were purchased from Fcmacs (cat. no. FMS-ABPE0001).

Cell culture and differentiation. The HL-60 cell line was obtained from the American Type Culture Collection. Cells were cultured in RPMI-1640 medium supplemented with $10 \%$ FBS or X-VIVO ${ }^{\text {TM }} 15$ medium in a humidified atmosphere containing $5 \% \mathrm{CO}_{2}$ at $37^{\circ} \mathrm{C}$. Cells were passaged every three days and only cells passaged no more than 15 times were used for the experiments. ATRA $(1 \mu \mathrm{mol} / \mathrm{l})$ or DMSO (1.25\%) were used to induce HL-60 cells to differentiate. CD11b, CD16 and CD66b expression was detected to evaluate the rate of HL-60 differentiation using a BD FACSCanto II flow cytometer (BD Biosciences). FITC Annexin V Apoptosis Detection kits were used to determine the viability of differentiated cells using a BD FACSCanto II flow cytometer (BD Biosciences).

Quantification of ROS production using flow cytometry. Total ROS Assay kits were used to quantify ROS in cells. The 500 X ROS Assay Stain stock solution was diluted to $1 \mathrm{X}$ using the ROS Assay Buffer. The cells were centrifuged for $5 \mathrm{~min}$ at $350 \mathrm{x}$ g and washed with PBS. Washed cells $\left(1 \times 10^{6}\right.$ cells $\left./ \mathrm{ml}\right)$ were incubated with $1 \mathrm{X}$ ROS assay stain for $60 \mathrm{~min}$ in a $37^{\circ} \mathrm{C}$ incubator with $5 \% \mathrm{CO}_{2}$, followed by treatment with phorbol 12-myristate 13 -acetate (PMA; $50 \mathrm{nmol} / \mathrm{l})$ or CI $(4 \mu \mathrm{mol} / \mathrm{l})$ for $1.5 \mathrm{~h}$ to induce the production of ROS. The analysis was performed with a BD FACSCanto II flow cytometer using the $488 \mathrm{~nm}$ (blue) laser in the FITC channel.

NETs visualization by fluorescence microscopy. In order to avoid abnormal NET generation caused by serum, the dHL-60 cells were centrifuged for $5 \mathrm{~min}$ at $350 \mathrm{xg}$ at $4^{\circ} \mathrm{C}$ and washed with PBS. Washed cells $\left(5 \times 10^{5}\right.$ cells $\left./ \mathrm{ml}\right)$ were incubated for $1 \mathrm{~h}$ in RPMI-1640 in confocal dishes and the cells were then stimulated with $50 \mathrm{nmol} / 1$ of PMA or $4 \mu \mathrm{mol} / 1$ of CI for $4 \mathrm{~h}$ in a humidified atmosphere containing $5 \% \mathrm{CO}_{2}$ at $37^{\circ} \mathrm{C}$. Cells were fixed with $4 \%$ paraformaldehyde for $20 \mathrm{~min}$ at room temperature and permeabilized with $0.3 \%$ Triton $\mathrm{X}-100$ for $10 \mathrm{~min}$ at room temperature. Samples were blocked with $10 \%$ goat serum for $1 \mathrm{~h}$ at $37^{\circ} \mathrm{C}$ and then incubated with antibodies directed against MPO (1:100 dilution) or H3 (1:100 dilution) overnight at $4^{\circ} \mathrm{C}$. After washing with PBS, the cells were incubated with goat anti-rabbit Alexa Fluor 568 (1:1,000 dilution) and goat anti-mouse Alexa Fluor 488 (1:500 dilution) for $1 \mathrm{~h}$ at $37^{\circ} \mathrm{C}$ in the dark. Cells were incubated with DAPI for $10 \mathrm{~min}$ at room temperature and exposed to ProLong Gold Antifade reagent according to the manufacturer's protocols. Cells were visualized using a confocal TCS SP8 microscope (Leica Microsystems).

Comparison of NETs composition. Cells were visualized using a confocal microscope and the composition of NETs was calculated via four random fluorescence scanning planes using ImageJ software (version 1.46r; National Institute of Health) (6). The average size of individual NETs was calculated by measuring the area of DNA stained by DAPI, the average size of NET-associated proteins was calculated from the area of MPO or histone H3. The composition of NETs was determined by calculating the average size of NET-associated proteins divided by the average size of the area occupied by DNA.

Quantification of NETs. PicoGreen was used to detect dsDNA following the manufacturer's protocol. Washed dHL-60 cells were transferred to 96 -well plates $\left(1 \times 10^{6}\right.$ cells $/ \mathrm{ml}$ in RPMI-1640) and the cells were stimulated with $50 \mathrm{nmol} / 1 \mathrm{PMA}$ or $4 \mu \mathrm{mol} / \mathrm{l} \mathrm{CI}$ for $4 \mathrm{~h}$. The supernatant was gently removed and replenished with $100 \mu \mathrm{l}$ PBS and an equivalent amount of 1X Quant-iT PicoGreen reagent was added to the 96-well plates, followed by incubation for $5 \mathrm{~min}$ at room temperature. Fluorescence was detected at an excitation wavelength 
of $502 \mathrm{~nm}$ with an emission wavelength of $523 \mathrm{~nm}$ using a spectrofluorometer (SpectraMax M2; Molecular Devices).

CitH3 quantification by flow cytometry. CitH3 quantification was performed as previously described (19), with certain modifications. Anti-citH3 antibody was pre-conjugated with PE-antibody using antibody conjugation kits. Cells were cultured in RPMI-1640 and stimulated with PMA (50 nmol/l) or $\mathrm{CI}(4 \mu \mathrm{mol} / \mathrm{l})$ for $2 \mathrm{~h}$ at $37^{\circ} \mathrm{C}$ in $5 \% \mathrm{CO}_{2}$, fixed in $2 \%$ paraformaldehyde for $20 \mathrm{~min}$, washed with PBS and centrifuged for $20 \mathrm{~min}$ at $16,000 \mathrm{x} \mathrm{g}$ at $4^{\circ} \mathrm{C}$. Cells were incubated with citH3-PE for $30 \mathrm{~min}$ at room temperature in the dark, washed with PBS, centrifuged for $20 \mathrm{~min}$ at $16,000 \times \mathrm{g}$ at $4^{\circ} \mathrm{C}$ and analyzed using a BD FACSCanto II flow cytometer.

Statistical analysis. SPSS Statistics 23.0 for Win10 (IBM Corp.) was used for data processing. Normally distributed data were expressed as the mean \pm standard deviation. One-way ANOVA was used to analyze differences among groups. As a multiple-comparisons test, Dunnett's test was selected if one column represented control data and Tukey's test was chosen when comparing all pairs of columns. Two-way ANOVA with Bonferroni's test for multiple comparisons was used when there were two independent variables. $\mathrm{P}<0.05$ was considered to indicate a statistically significant difference.

\section{Results}

Differentiation and apoptosis of HL-60 cells under ATRA and $D M S O$. Since the HL-60 differentiation time was different in previous studies $(13,15,20-22)$, the present study aimed to determine the differentiation protocol that led to the highest expression of the neutrophil surface marker, while maintaining high cell viability. The differentiation was observed for three to seven days using the most commonly used differentiation agents, ATRA and DMSO. High concentrations of serum have been reported to inhibit differentiation (23). X-VIVO ${ }^{\text {Tм }} 15$ is a serum-free medium, which has been reported to improve cell differentiation (24). Hence, the effects of serum (RPMI-1640 supplemented with $10 \%$ FBS) and serum-free medium (X-VIVO ${ }^{\mathrm{TM}} 15$ medium) on the differentiation of HL-60 cells were also compared.

To investigate the differentiation efficiency, CD11b, CD16 and CD66b were used as the neutrophil surface markers $(20,25-27)$ and Apoptosis Detection kits were used for the evaluation of cell viability. Both ATRA and DMSO need five days to produce efficient expression of CD11b, CD16 and CD66b in dHL-60 cells incubated in normal medium with serum or X-VIVO 15 medium (Fig. 1A-N). With increasing differentiation time, the expression of CD11b, CD16 and CD66b decreased in certain groups (Fig. 1M-O). The association between the differentiation time and the survival rate of cells was determined and the results indicated that the cell viability was similar from the third to the fifth day; however, cell death increased significantly from the sixth day (Fig. 1P).

Since induction for five days produced the best results in terms of differentiation markers and cell viability, the expression of CD11b, CD16, and CD66b was compared in different groups. The group with the best differentiation potential [DMSO-dHL-60 (FBS)] was used as a representative control group. It was observed that CD11b expression was significantly higher in the DMSO-dHL-60 (FBS) group than in the ATRA-dHL-60 (X-VIVO) group (Fig. 1M). The expression of CD66b was also significantly higher in the DMSOdHL-60 (FBS)group as compared with that in the ATRA-dHL-60 (FBS) group (Fig. 1N). After induction for 5 days, the DMSO-dHL-60 (FBS) group had the highest expression of CD16 among the groups (Fig. 1O).

In summary, induction for five days provided the best results in terms of differentiation markers and cell viability, while among the four methods studied, the differentiation protocol involving the induction of differentiation using DMSO in serum-containing medium produced the best differentiation potential.

ROS formation in dHL-60 cells after stimulation with PMA or $C I$. In the above experiments, it was determined that induction for five days produced the best results in terms of differentiation markers and cell viability. In subsequent experiments, the ability of dHL-60 cells to produce ROS after five days of differentiation was tested. ROS formation was analyzed using Total Reactive Oxygen Species Assay kits, which contain the necessary reagents and buffers for identifying ROS in cells by flow cytometry in the FITC channel. It was determined that all four different protocols may induce HL-60 cells to produce ROS upon stimulation with PMA (Fig. 2A and C). Both ATRA-dHL-60 and DMSO-dHL-60 cells cultured in serum-free medium released more ROS than the cells in serum-containing medium. DMSO-dHL-60 (X-VIVO) was the most efficient of the four groups (Fig. 2C). Under stimulation with CI, the ATRA-dHL-60 (FBS) group was not able to form ROS. ROS formation was more effective in serum-free medium than in serum-containing medium with the same agents and ATRA-dHL-60 (X-VIVO) was the most efficient of all four groups (Fig. 2B and D).

Stimulation of differentiated HL-60 cells in serum-free medium results in highly efficient NETs production. In the above experiments, it was determined that ROS formation was more effective in serum-free medium than in serum-containing medium. As the release of NETs occurs downstream of ROS generation, the ability of dHL-60 cells to produce NETs after stimulation was tested in subsequent experiments. By fluorescent microscopy, it was observed that all four differentiation protocols are able to release NETs upon stimulation by both PMA and CI (Fig. 3A).

Quantification of NETs using PicoGreen indicated that these cells release DNA when stimulated with PMA or CI; however, differentiated HL-60 cells in serum-free medium appeared to produce more NETs than those in serum-containing medium (Fig. 3B). Therefore, the numerical value of NETs was compared according to the medium in which the cells were incubated. The results indicated that both ATRA and DMSO dHL-60 cells in serum-free medium formed more NETs than those in serum-containing medium upon stimulation with PMA or CI. The DMSO-dHL-60 (X-VIVO) group was the most efficient in releasing NETs post-PMA stimulation, while the ATRA-dHL-60 (X-VIVO) group was the most efficient in releasing NETs post-CI stimulation (Fig. 3C). 
A

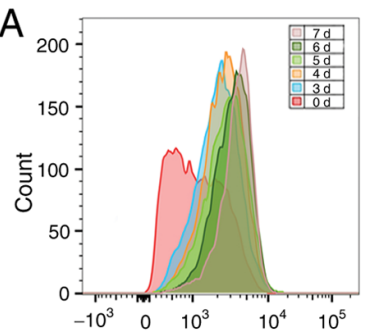

CD11b-PE-ATRA-dHL-60 (FBS)

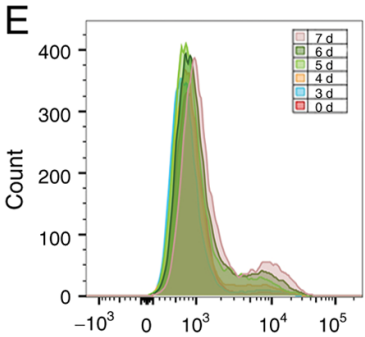

CD66b-FITC-ATRA-dHL-60 (FBS)

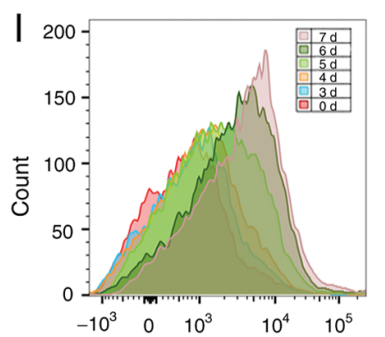

$\mathrm{M}$

CD16-APC-ATRA-dHL-60 (FBS)

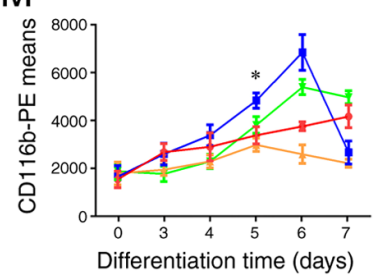

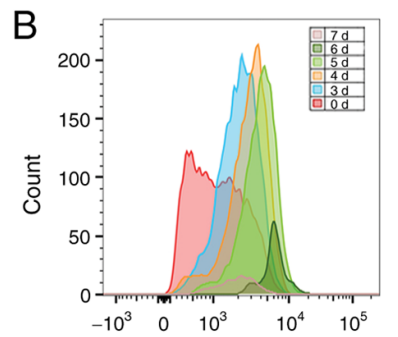

CD11b-PE-DMSO-dHL-60 (FBS)

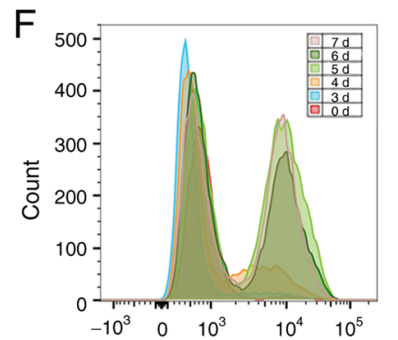

CD66b-FITC-DMSO-dHL-60 (FBS)

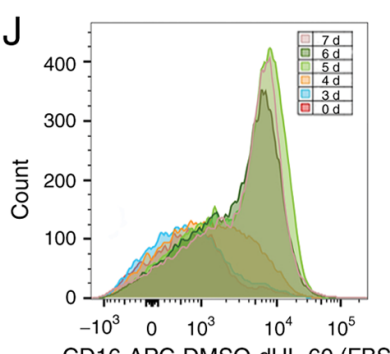

$\mathrm{N}$
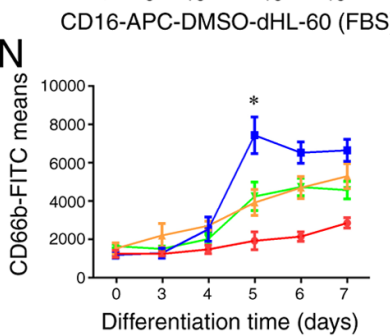

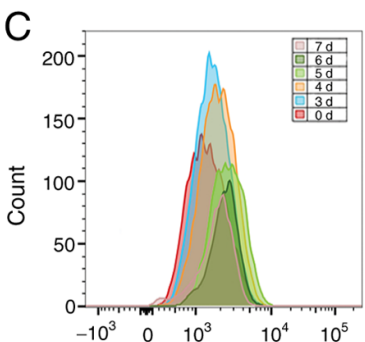

CD11b-PE-ATRA-dHL-60 (X-VIVO)

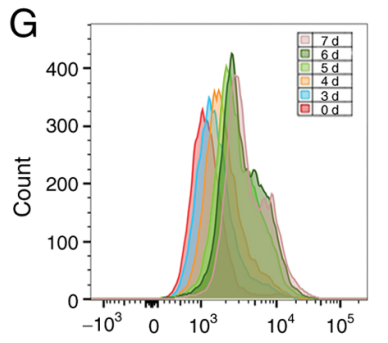

CD66b-FITC-ATRA-dHL-60 (X-VIVO)

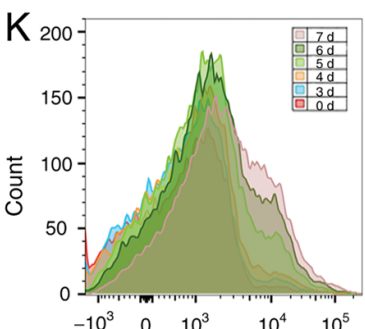

0

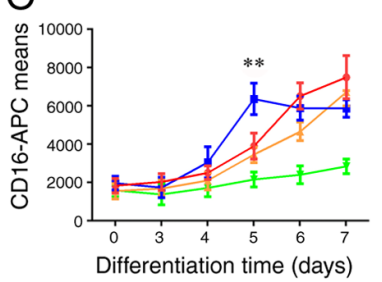

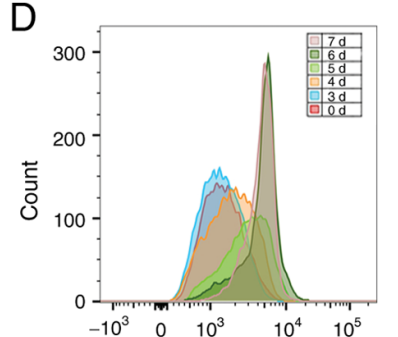

CD11b-PE-DMSO-dHL-60 (X-VIVO)

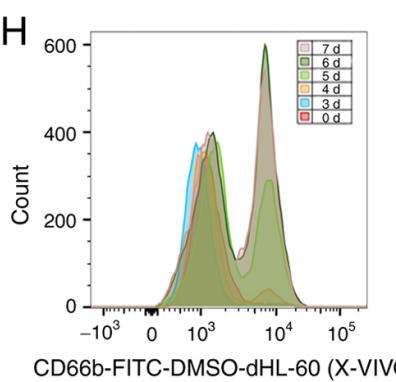

- ATRA-HL-60 (FBS)
- ATRA-HL-60 (X-VIVO) - ATRA-HL-60 (FBS)

$\rightarrow$ ATRA-HL-60 (FBS) $\rightarrow$ ATRA-HL-60 (X-VIVO)

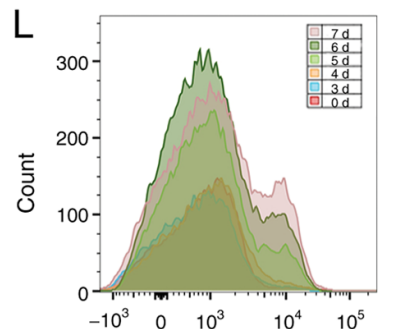

$P$

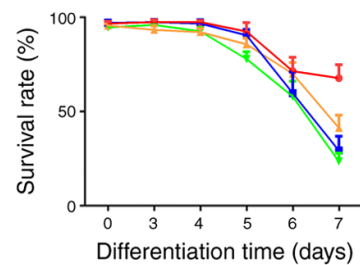

$\rightarrow$ ATRA-HL-60 (FBS) $\#$ ATRA-HL-60 (X-VIVO)

Figure 1. Differentiation and apoptosis of HL-60 cells in the presence of ATRA and DMSO. HL-60 cells were differentiated into a neutrophil-like state by stimulation with ATRA $(1 \mu \mathrm{mol} / \mathrm{l})$ or DMSO (1.25\%) for three to seven days in serum-containing medium (RPMI-1640 supplemented with $10 \%$ FBS) or serum replacement medium (X-VIVO ${ }^{\text {TM }} 15$ Medium). (A-D) CD11b, (E-H) CD16 and (I-L) CD66b expression was used as a neutrophil surface marker to measure the differentiation state in (A, E, I) ATRA-dHL-60 (FBS), (B, F and J) DMSO-dHL-60 (FBS), (C, G and K) ATRA-dHL-60 (X-VIVO) and (D, H and L) DMSO-dHL-60 (X-VIVO). (M-O) CD11b, CD66b and CD16 expression was compared between the four protocols during three to seven days of differentiation; one-way ANOVA with Dunnett's test for multiple comparisons was used for comparing the expression of surface markers on the fifth differentiation day. (M) CD11b expression in was significantly higher in the DMSO-dHL-60 (FBS) group than in the ATRA-dHL-60 (X-VIVO) group, ${ }^{*}<0.05$. (N) The expression of CD66b was also significantly higher in the DMSO-dHL-60 (FBS) group compared with that in the ATRA-dHL-60 (FBS) group, ${ }^{*}<0.05$. (O) The DMSO-dHL-60 (FBS) group had the highest expression of CD16 among the groups; ${ }^{* *} \mathrm{P}<0.01$. (P) Cell viability was similar from the third day to the fifth day and decreased significantly from the sixth day. Samples were measured using flow cytometry and data were analyzed using FlowJo software. The experiments were performed three times and a representative experiment is presented. ATRA, all-trans retinoic acid; DMSO, dimethyl sulfoxide; dHL-60, differentiated HL-60 cells; d, days.

Next, the composition of NETs was compared by calculating the areas of NET-associated proteins (MPO and histone H3) divided by DNA areas determined using fluorescent microscopy (6). When dHL-60 cells were stimulated with PMA, both ATRA and DMSO dHL-60 groups in serum-free medium released a greater ratio of histone $\mathrm{H} 3$ to DNA than those in serum-containing medium (Fig. 3D); however, the ratio of MPO expression exhibited no difference. There was no statistically significant difference in the composition of NET-associated proteins between the groups upon CI stimulation (Fig. 3E). Furthermore, the composition of
NET-associated proteins differed for different stimulation agents (data not shown).

Histone citrullination in $d H L-60$ cells after stimulation with PMA or CI. The conversion of arginine into citrulline by PADI4 may contribute to chromatin relaxation, which is frequently required for NET extrusion via suicidal and vital NETosis (28). To evaluate the ability for histone citrullination in different protocols after stimulation, the formation of citH3 was detected using a flow cytometer. The results indicated that none of the dHL-60 cells contained citH3 upon PMA 
A
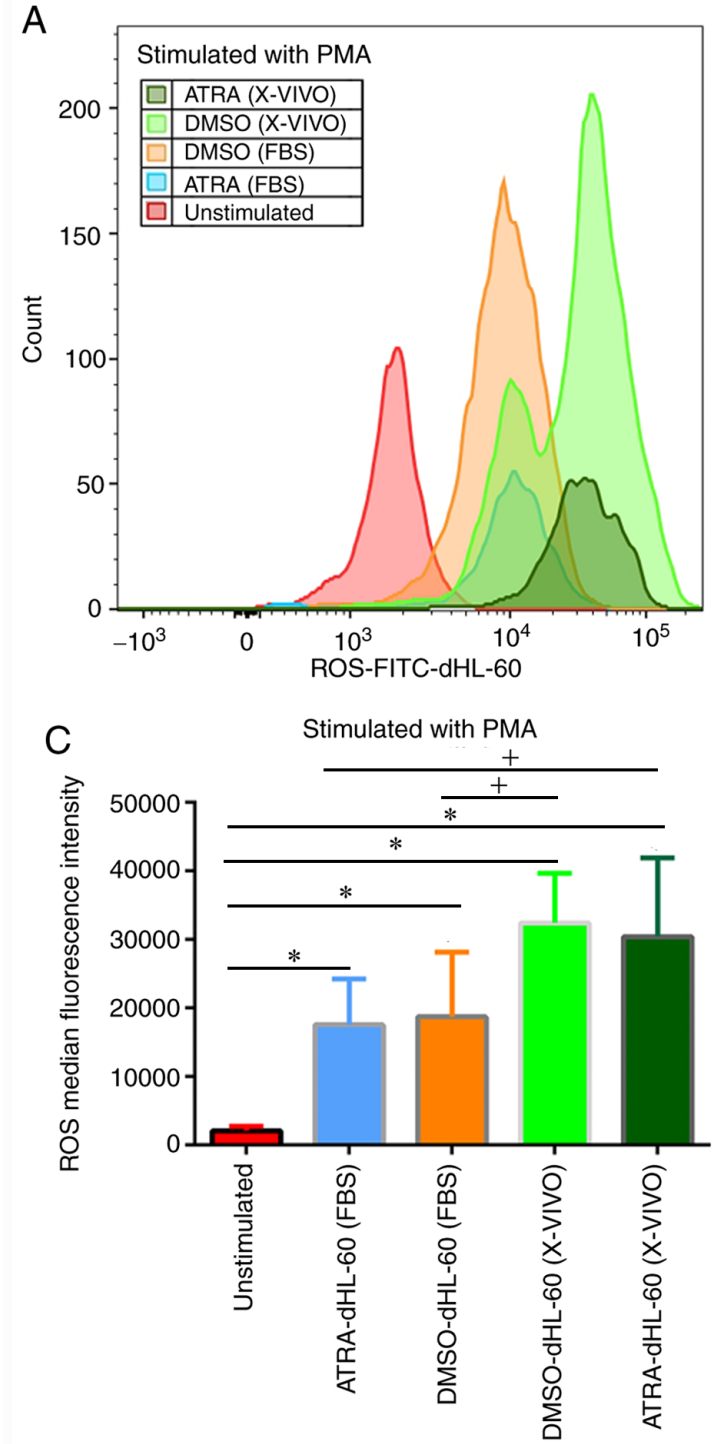

B

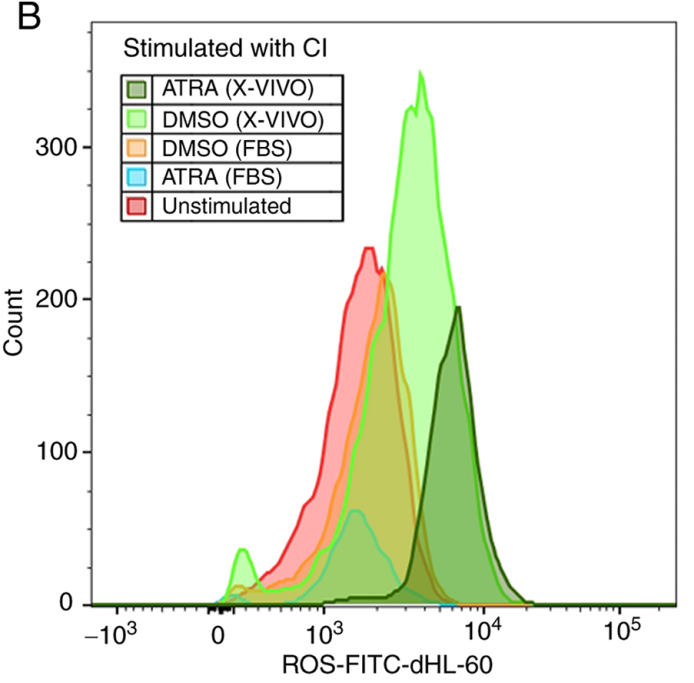

D

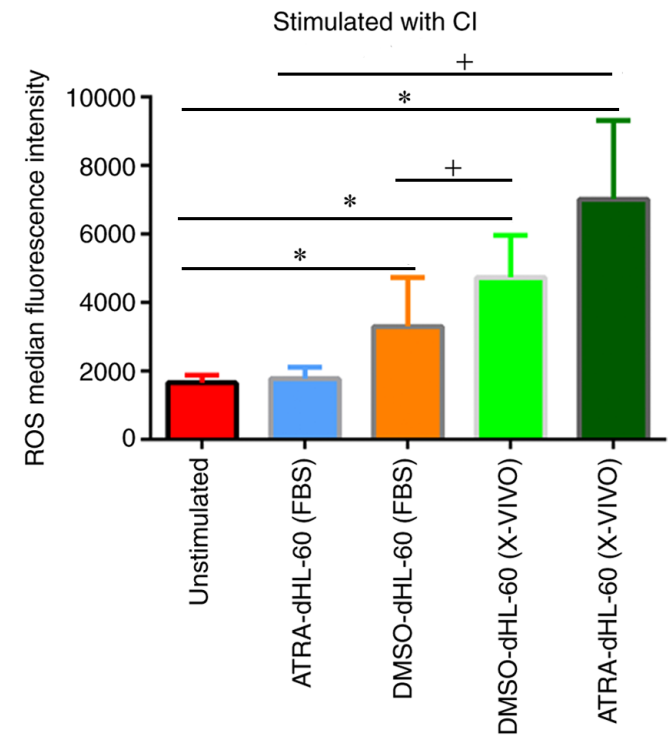

Figure 2. ROS formation in dHL-60 cells after stimulation. (A and B) ROS formation stimulated with (A) PMA or (B) CI was assessed by flow cytometry in the FITC channel. (C and D) Median fluorescence intensity of ROS stimulated with (C) PMA or (D) CI. Values are expressed as the mean \pm standard error of the mean calculated from three independent experiments; one-way ANOVA with Dunnett's test for multiple comparisons was used for comparing the expression between experimental groups and control group; ${ }^{*} \mathrm{P}<0.05$. One-way ANOVA with Tukey's test for multiple comparisons was used for comparing the expression between the serum-free group and serum-containing group with the same differentiation agent; ${ }^{+} \mathrm{P}<0.05$. ROS, reactive oxygen species; $\mathrm{dHL}-60$, differentiated HL-60 cells; ATRA, all-trans retinoic acid; DMSO, dimethyl sulfoxide; PMA, phorbol 12-myristate 13-acetate; CI, Ca ${ }^{2+}$ ionophore.

treatment (Fig. 4A and C). After CI treatment, the proportion of citH3-positive cells among dHL-60 cells was $10.4 \%$ in the ATRA-dHL-60 (FBS), 6.58\% in the DMSO-dHL-60 (FBS), $32.8 \%$ in the ATRA-dHL-60 (X-VIVO) and $18.4 \%$ in the DMSO-dHL-60 (X-VIVO) group; however, ATRA-dHL-60 (X-VIVO) was the most effective group regarding citH3 formation after CI stimulation (Fig. 4B and C).

\section{Discussion}

In the present study, the ability of dHL-60 cells to form NETs under different differentiation conditions and with different stimulation agents was assessed. The experimental evidence suggested that the optimal experimental protocol involved differentiation of HL-60 cells for five days and culture in serum-free medium. With respect to the ability of dHL-60 cells to release NETs, DMSO-dHL-60 (X-VIVO) cells were most efficient at producing NETs and ROS upon PMA stimulation, while ATRA-dHL-60 (X-VIVO) cells were most efficient at producing NETs and citH3 upon CI stimulation.

The biology of neutrophils remains to be fully elucidated and the short life span of neutrophils makes it difficult to study their functions. HL-60 cells are a human myeloid leukemia cell line, which may undergo granulocytic differentiation after stimulation using agents such as ATRA and DMSO (13,29-33). There are various differentiation conditions. For stimulation using DMSO, Song et al (32) and Wang et al (34) treated HL-60 cells for three days, Gupta et al (22) suggested incubation for three to four days, Manda-Handzlik et al (13) treated the cells for five days and Razvina et al (16) treated the cells for seven days. The treatment time with ATRA was also different in previous studies $(13,20,22,32)$. Since neutrophilic differentiation is frequently coordinated with cell death, exploration of HL-60 differentiation conditions is conducive to effective 

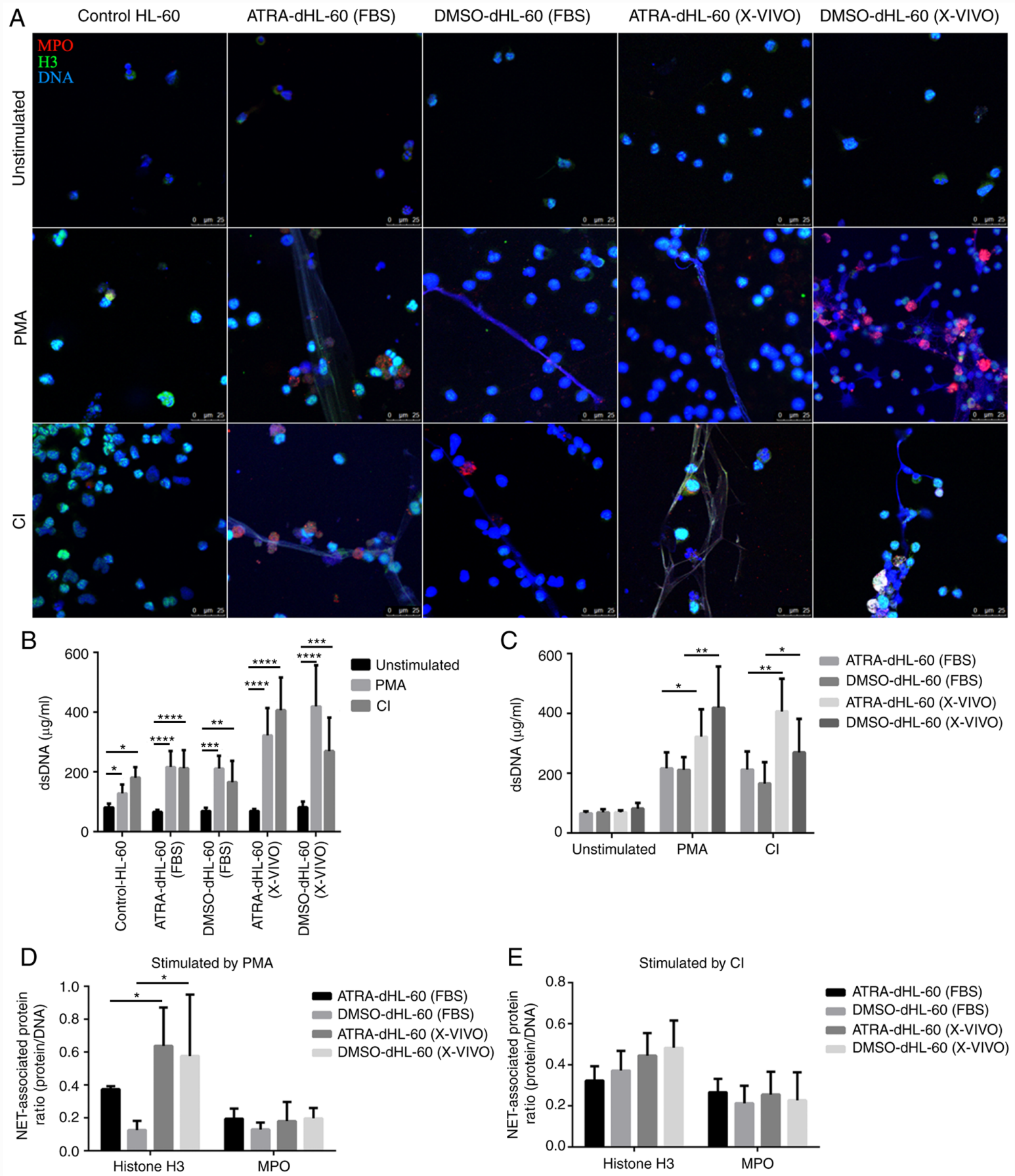

Figure 3. NETs released from dHL-60 post-PMA and CI stimulation. (A) NETs formation was detected by fluorescent microscopy. Samples were stained with antibodies against MPO (red) and histone H3 (green). DNA was counterstained with DAPI (original magnification, x20; scale bar, $25 \mu \mathrm{m}$ ). (B and C) DNA release was detected by PicoGreen. (B) One-way ANOVA with Dunnett's test for multiple comparisons was used for comparing the DNA release between the stimulated and unstimulated groups. All four different protocols can induce HL-60 cells to produce NETs upon stimulation with PMA and CI. "P<0.05, ${ }^{* *} \mathrm{P}<0.01,{ }^{* * * *} \mathrm{P}<0.001$ and ${ }^{* * * * *} \mathrm{P}<0.0001$. (C) One-way ANOVA with Tukey's test for multiple comparisons was used for comparing the DNA release between the serum-containing medium group and the serum-free medium group with the group with the same differentiation agents, ${ }^{*} \mathrm{P}<0.05$, ${ }^{* * *} \mathrm{P}<0.01$. In $\mathrm{B}$ and $\mathrm{C}$, values are expressed as the mean \pm standard error of the mean from six independent experiments. (D and E) The composition of NETs was detected by calculating the areas of NET-associated proteins (MPO and histone H3) divided by DNA area. (D) Stimulation with PMA and compared between the serum-containing medium group and serum-free medium group with the same differentiation agents, ${ }^{\text {" }} \mathrm{P}<0.05$. (E) Stimulation with $\mathrm{CI}$ and compared between the serum-containing medium group and serum-free medium group with the same differentiation agents. Data were measured using ImageJ software. NETs, neutrophil extracellular traps; MPO, myeloperoxidase; dHL-60, differentiated HL-60 cells; ATRA, all-trans retinoic acid; DMSO, dimethyl sulfoxide; PMA, phorbol 12-myristate 13-acetate; CI, $\mathrm{Ca}^{2+}$ ionophore; dsDNA, double-strand DNA.

differentiation. The present results indicated that incubation with DMSO or ATRA for five days was the most suitable period for inducing neutrophil marker expression and cell viability.
NETs have critical roles in the host defense mechanism mediated by neutrophils; therefore, numerous studies have used dHL-60 to explore the molecular and cellular mechanisms of NETs formation $(14,16,17,29)$. However, the capacity 
A
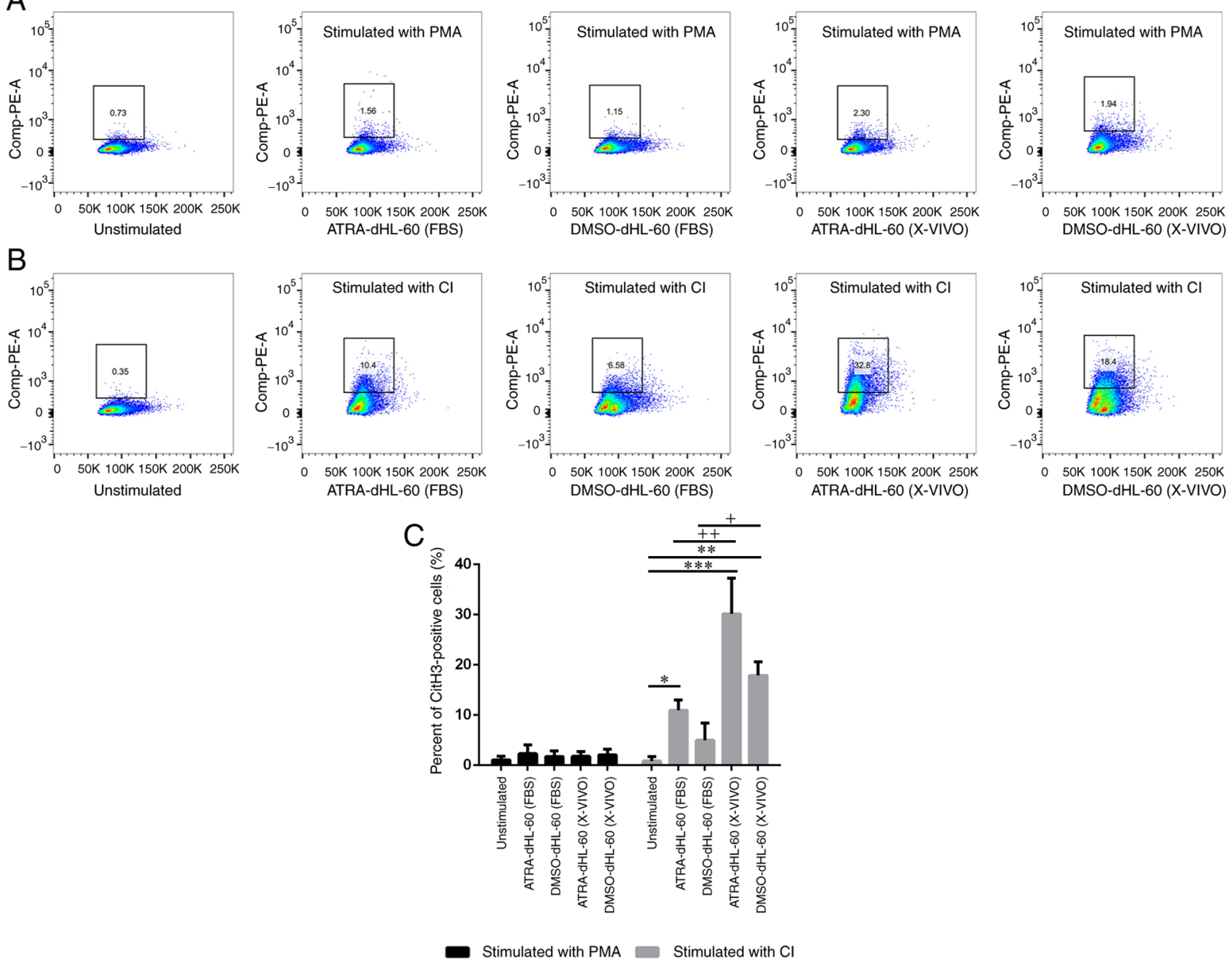

Figure 4. Histone citrullination in dHL-60 cells after stimulation. (A and B) Representative flow cytometry images for the identification of citH3-positive cells defined as NETs. (A) Stimulation with PMA and (B) stimulation with CI. (C) Two-way ANOVA with Bonferroni's test for multiple comparisons was used for comparing the percentage of citH3-positive cells among the groups. After stimulation with CI, the percentage of citH3-positive cells was significantly higher in ATRA-dHL-60 (FBS), ATRA-dHL-60 (X-VIVO) and DMSO-dHL-60 (X-VIVO) groups as compared with that in unstimulated group, "P<0.05 ${ }^{* *} \mathrm{P}<0.01$ and ${ }^{* * *} \mathrm{P}<0.001$. The percentage of citH3-positive cells was significantly higher in serum-free group as compared with that in serum-containing group [DMSO-dHL-60 (X-VIVO) vs. DMSO-dHL-60 (FBS); ${ }^{+} \mathrm{P}<0.05$ and ATRA-dHL-60 (X-VIVO) vs. ATRA-dHL-60 (FBS), $\left.{ }^{++} \mathrm{P}<0.01\right]$ when stimulation with CI. NETs, neutrophil extracellular traps; dHL-60, differentiated HL-60 cells; ATRA, all-trans retinoic acid; DMSO, dimethyl sulfoxide; PMA, phorbol 12-myris tate 13-acetate; $\mathrm{CI}, \mathrm{Ca}^{2+}$ ionophore; citH3, citrullinated histone $\mathrm{H} 3$.

of dHL-60 to release NETs was weak compared with that of neutrophils $(13,30)$. A maximum of $28 \%$ of NETs release was reached after $4 \mathrm{~h}$ of co-incubation of dHL-60 with PMA, while blood-derived neutrophils produced almost $100 \%$ NETs after $4 \mathrm{~h}$ of stimulation (30). It is important to increase the ability of dHL-60 cells to release NETs. High concentrations of serum have been reported to inhibit differentiation (23). To suppress the adverse effects of serum, a serum-free medium (X-VIVO ${ }^{\text {Tм }} 15$ medium), which has been reported to improve cell differentiation (24), was used in the present study. The present results confirmed that the ability of dHL-60 cells cultured in serum-free media to release NETs upon PMA or CI stimulation was greater than that of cells cultured in serum-containing media.

PMA and CI are commonly used for the study of NETosis. PMA is able to activate numerous pathways in neutrophils and mediate suicidal NETosis. Upon treatment with PMA, NADPH oxidase is activated via protein kinase $\mathrm{C}$ and the Raf-MAPK kinase-ERK signaling pathway, leading to ROS generation and
MPO activation and resulting in chromatin decondensation in the nucleus $(7,35)$. In this cell death process, the generation of ROS by NADPH oxidase is critical. Consistent with the weak ability of dHL-60 cells to release NETs, they do not release ROS as vigorously as neutrophils $(29,30,36)$. Imaizumi and Breitman (33) reviewed several studies and concluded that the capacity for ROS production by DMSO-dHL-60 cells was stronger than that of ATRA-dHL-60 cells upon PMA stimulation. Sham et al (31) reported the opposite result. In the present study, it was observed that the capacity for ROS production was similar between DMSO-dHL-60 (FBS) cells and ATRA-dHL-60 (FBS) cells; however, ROS formation was more effective in serum-free medium than in serum-containing medium with the same agents and DMSO-dHL-60 (X-VIVO) was the most effective.

Neutrophils may form NETs independent of oxidant production. CI-induced NET formation is dependent on AKT signaling and calcium-induced mitochondrial ROS, but not on the NADPH oxidase pathway $(18,37)$. The present study also determined that 
the ROS release stimulated by CI was much lower than with PMA. Serum may influence the generation of ROS and NET formation (38) and the presence of DNase I in serum also affects the degradation of NETs (39). To avoid any interference of serum with ROS and NET generation, all dHL-60 cells cultured in media were washed and cultured with RPMI-1640 during stimulation.

The formation of NETs is a process dependent on both ROS formation and histone citrullination. It was indicated that dHL-60 cells only exhibited histone citrullination upon CI stimulation but not upon PMA stimulation. This result was consistent with the results of previous neutrophil stimulation experiments using CI and PMA (18). The present study indicated that citH3 formation after CI stimulation was highest in the ATRA-dHL-60 (X-VIVO) group. Song et al (32) reported that ATRA stimulation led to significant upregulation of PADI4 expression at both the mRNA and protein levels in HL-60 cells in a time-dependent manner. It was speculated that the effective histone citrullination ability of ATRA-dHL-60 cells may be due to the upregulation of PADI4.

In conclusion, the optimal experimental protocol for HL-60 cells was differentiation for five days and culture in serum-free medium. The DMSO-dHL-60 (X-VIVO) group was most efficient in terms of NETs and ROS production upon PMA stimulation, while the ATRA-dHL-60 (X-VIVO) group was most efficient in terms of NETs and citH3 generation upon CI stimulation. Based on the different pathways of PMA and CI, DMSO-dHL-60 (X-VIVO) may be proposed as a model for the study of ROS-high NETosis, while ATRA-dHL-60 (X-VIVO) may be suitable for ROS-low NETosis.

\section{Acknowledgements}

The authors would like to thank Mrs Ying Yin (Department of Central Laboratory, Wuxi People's Hospital Affiliated to Nanjing Medical University, Wuxi, China) for performing the flow cytometry detection and Miss Jianxin Tan (Department of Central Laboratory, Wuxi People's Hospital Affiliated to Nanjing Medical University, Wuxi, China) for acquiring laser confocal microscopy images.

\section{Funding}

This study was funded by the Medical Innovation Team of Jiangsu Province (grant no. CXTDB 2017016), the General Program of Nanjing Medical University (grant no. 2017NJMUZD119), Wuxi Municipal Bureau on Science and Technology (grant no. NZ2019026), the Young Project of Wuxi Health and Family Planning Commission (grant no. Q202054) and the Major Program of Wuxi Health and Family Planning Commission (grant no. Z202016).

\section{Availability of data and materials}

The datasets used and/or analyzed during the current study are available from the corresponding author on reasonable request.

\section{Authors' contributions}

LL and DZ designed, supervised the present study and can authenticate the raw data in the study. YG and FG performed the experiments and were involved in writing the manuscript. QW performed quality control and adjustment on the flow cytometry experiment. KW conducted cell culture and differentiation. SP analyzed the flow cytometry results. ZP completed the statistical analyzes of all results. SX analyzed the fluorescence microscopy data. All authors read and approved the final manuscript.

\section{Ethics approval and consent to participate}

Not applicable.

\section{Patient consent for publication}

Not applicable.

\section{Competing interests}

The authors declare that they have no competing interests.

\section{References}

1. Soehnlein O, Steffens S, Hidalgo A and Weber C: Neutrophils as protagonists and targets in chronic inflammation. Nat Rev Immunol 17: 248-261, 2017.

2. Castanheira FVS and Kubes P: Neutrophils and NETS in modulating acute and chronic inflammation. Blood 133: 2178-2185, 2019.

3. Lefrancais E, Mallavia B, Zhuo H, Calfee CS and Looney MR: Maladaptive role of neutrophil extracellular traps in pathogeninduced lung injury. JCI Insight 3: e98178, 2018.

4. Mohanty T, Fisher J, Bakochi A, Neumann A, Cardoso JFP, Karlsson CAQ, Pavan C, Lundgaard I, Nilson B, Reinstrup P, et al: Neutrophil extracellular traps in the central nervous system hinder bacterial clearance during pneumococcal meningitis. Nat Commun 10: 1667, 2019.

5. Tanaka M, Kinoshita-Daitoku R, Kiga K, Sanada T, Zhu B, Okano T, Aikawa C, Iida T, Ogura Y, Hayashi T, et al: Group A streptococcus establishes pharynx infection by degrading the deoxyribonucleic acid of neutrophil extracellular traps. Sci Rep 10: 3251, 2020.

6. van Dam LS, Kraaij T, Kamerling SWA, Bakker JA, Scherer UH, Rabelink TJ, van Kooten C and Teng YKO: Intrinsically distinct role of neutrophil extracellular trap formation in antineutrophil cytoplasmic antibody-associated vasculitis compared to systemic lupus erythematosus. Arthritis Rheumatol 71: 2047-2058, 2019.

7. Brinkmann V, Reichard U, Goosmann C, Fauler B, Uhlemann Y, Weiss DS, Weinrauch Y and Zychlinsky A: Neutrophil extracellular traps kill bacteria. Science 303: 1532-1535, 2004.

8. Fuchs TA, Abed U, Goosmann C, Hurwitz R, Schulze I, Wahn V, Weinrauch Y, Brinkmann V and Zychlinsky A: Novel cell death program leads to neutrophil extracellular traps. J Cell Biol 176: 231-241, 2007.

9. D'Cruz AA, Speir M, Bliss-Moreau M, Dietrich S, Wang S, Chen AA, Gavillet M, Al-Obeidi A, Lawlor KE and Vince JE: The pseudokinase MLKL activates PAD4-dependent NET formation in necroptotic neutrophils. Sci Signal 11: eaao1716, 2018.

10. Yousefi S, Mihalache C, Kozlowski E, Schmid I and Simon HU: Viable neutrophils release mitochondrial DNA to form neutrophil extracellular traps. Cell Death Differ 16: 1438-1444, 2009.

11. Pilsczek FH, Salina D, Poon KK, Fahey C, Yipp BG, Sibley CD, Robbins SM, Green FH, Surette MG, Sugai M, et al: A novel mechanism of rapid nuclear neutrophil extracellular trap formation in response to Staphylococcus aureus. J Immunol 185: 7413-7425, 2010.

12. Chen KW, Monteleone M, Boucher D, Sollberger G, Ramnath D, Condon ND, von Pein JB, Broz P, Sweet MJ and Schroder K: Noncanonical inflammasome signaling elicits gasdermin D-dependent neutrophil extracellular traps. Sci Immunol 3: eaar6676, 2018. 
13. Manda-Handzlik A, Bystrzycka W, Wachowska M, Sieczkowska S, Stelmaszczyk-Emmel A, Demkow U and Ciepiela O: The influence of agents differentiating HL-60 cells toward granulocyte-like cells on their ability to release neutrophil extracellular traps. Immunol Cell Biol 96: 413-425, 2018.

14. Manda-HandzlikA,BystrzyckaW,CielochA,Glodkowska-MrowkaE, Jankowska-Steifer E, Heropolitanska-Pliszka E, Skrobot A, Muchowicz A, Ciepiela O, Wachowska M and Demkow U: Nitric oxide and peroxynitrite trigger and enhance release of neutrophil extracellular traps. Cell Mol Life Sci 77: 3059-3075, 2020.

15. Obama T, Ohinata H, Takaki T, Iwamoto S, Sawada N, Aiuchi T, Kato R and Itabe H: Cooperative action of oxidized low-density lipoproteins and neutrophils on endothelial inflammatory responses through neutrophil extracellular trap formation. Front Immunol 10: 1899, 2019.

16. Razvina O, Jiang S, Matsubara K, Ohashi R, Hasegawa G, Aoyama T, Daigo K, Kodama T, Hamakubo T and Naito $M$ Differential expression of pentraxin 3 in neutrophils. Exp Mol Pathol 98: 33-40, 2015.

17. Alyami HM, Finoti LS, Teixeira HS, Aljefri A, Kinane DF and Benakanakere MR: Role of NOD1/NOD2 receptors in Fusobacterium nucleatum mediated NETosis. Microb Pathog 131: 53-64, 2019.

18. Douda DN, Khan MA, Grasemann H and Palaniyar N: SK3 channel and mitochondrial ROS mediate NADPH oxidaseindependent NETosis induced by calcium influx. Proc Natl Acad Sci USA 112: 2817-2822, 2015.

19. Gavillet M, Martinod K, Renella R, Harris C, Shapiro NI, Wagner DD and Williams DA: Flow cytometric assay for direct quantification of neutrophil extracellular traps in blood samples Am J Hematol 90: 1155-1158, 2015

20. Rincon E, Rocha-Gregg BL and Collins SR: A map of gene expression in neutrophil-like cell lines. BMC Genomics 19: 573, 2018

21. Liu CL, Tangsombatvisit S, Rosenberg JM, Mandelbaum G, Gillespie EC, Gozani OP, Alizadeh AA and Utz PJ: Specific post-translational histone modifications of neutrophil extracellular traps as immunogens and potential targets of lupus autoantibodies. Arthritis Res Ther 14: R25, 2012.

22. Gupta D, Shah HP, Malu K, Berliner N and Gaines P: Differentiation and characterization of myeloid cells. Curr Protoc Immunol 104: 22F.5.1-22F.5.28, 2014.

23. Pedruzzi E, Fay M, Elbim C and Gougerot-Pocidalo MA: Differentiation of PLB-985 myeloid cells into mature neutrophils, shown by degranulation of terminally differentiated compartments in response to $\mathrm{N}$-formyl peptide and priming of superoxide anion production by granulocyte-macrophage colony-stimulating factor. Br J Haematol 117: 719-726, 2002.

24. Chen L, Xie XY, Nie JQ, Chen DL, Huang AP, Fang F, Qu MY, Nan X, He LJ, Fan Z, et al: Mononuclear cells of umbilical cord blood differentiation to granulocyte cell in vitro. Zhonghua Xue Ye Xue Za Zhi 38: 532-536, 2017 (In Chinese).

25. Perdomo J, Leung HH, Ahmadi Z, Yan F, Chong JJ, Passam FH and Chong BH: Neutrophil activation and NETosis are the major drivers of thrombosis in heparin-induced thrombocytopenia. Nat Commun 10: 1322, 2019.

26. Lelliott PM, Momota M, Shibahara T, Lee MS, Smith NI, Ishii KJ and Coban C: Heparin induces neutrophil elastase dependent vital and lytic NET formation. Int Immunol 32: 359-368, 2020.
27. Lood C, Blanco LP, Purmalek MM, Carmona-Rivera C, De Ravin SS, Smith CK, Malech HL, Ledbetter JA, Elkon KB and Kaplan MJ: Neutrophil extracellular traps enriched in oxidized mitochondrial DNA are interferogenic and contribute to lupus-like disease. Nat Med 22: 146-153, 2016.

28. Burgener SS and Schroder K: Neutrophil extracellular traps in host defense. Cold Spring Harb Perspect Biol 12: a037028, 2020.

29. Wang Y, Li M, Stadler S, Correll S, Li P, Wang D, Hayama R, Leonelli L, Han H, Grigoryev SA, et al: Histone hypercitrullination mediates chromatin decondensation and neutrophil extracellular trap formation. J Cell Biol 184: 205-213, 2009.

30. Yaseen R, Blodkamp S, Lüthje P, Reuner F, Völlger L, Naim HY and von Köckritz-Blickwede M: Antimicrobial activity of HL-60 cells compared to primary blood-derived neutrophils against Staphylococcus aureus. J Negat Results Biomed 16: 2 , 2017.

31. Sham RL, Phatak PD, Belanger KA and Packman CH: Functional properties of HL60 cells matured with all-trans-retinoic acid and DMSO: Differences in response to interleukin-8 and fMLP. Leuk Res 19: 1-6, 1995.

32. Song G, Shi L, Guo Y, Yu L, Wang L, Zhang X, Li L, Han Y, Ren X, Guo Q, et al: A novel PAD4/SOX4/PU.1 signaling pathway is involved in the committed differentiation of acute promyelocytic leukemia cells into granulocytic cells. Oncotarget 7: 3144-3157, 2016.

33. Imaizumi $M$ and Breitman TR: Retinoic acid-induced differentiation of the human promyelocytic leukemia cell line, HL-60, and fresh human leukemia cells in primary culture: A model for differentiation inducing therapy of leukemia. Eur J Haematol 38: 289-302, 1987.

34. Wang Y, Wysocka J, Sayegh J, Lee YH, Perlin JR, Leonelli L, Sonbuchner LS, McDonald CH, Cook RG, Dou Y, et al: Human PAD4 regulates histone arginine methylation levels via demethylimination. Science 306: 279-283, 2004.

35. Steinberg SF: Structural basis of protein kinase C isoform function. Physiol Rev 88: 1341-1378, 2008.

36. Remijsen Q, Vanden Berghe T, Wirawan E, Asselbergh B, Parthoens E, De Rycke R, Noppen S, Delforge M, Willems J and Vandenabeele P: Neutrophil extracellular trap cell death requires both autophagy and superoxide generation. Cell Res 21: 290-304, 2011.

37. Parker H, Dragunow M, Hampton MB, Kettle AJ and Winterbourn CC: Requirements for NADPH oxidase and myeloperoxidase in neutrophil extracellular trap formation differ depending on the stimulus. J Leukoc Biol 92: 841-849, 2012.

38. Kamoshida G, Kikuchi-Ueda T, Nishida S, Tansho-Nagakawa S, Kikuchi H, Ubagai T and Ono Y: Spontaneous formation of neutrophil extracellular traps in serum-free culture conditions. FEBS Open Bio 7: 877-886, 2017.

39. Hakkim A, Furnrohr BG, Amann K, Laube B, Abed UA, Brinkmann V, Herrmann M, Voll RE and Zychlinsky A: Impairment of neutrophil extracellular trap degradation is associated with lupus nephritis. Proc Natl Acad Sci USA 107: 9813-9818, 2010.

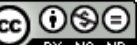

This work is licensed under a Creative Commons Attribution-NonCommercial-NoDerivatives 4.0 International (CC BY-NC-ND 4.0) License. 